\title{
ZAHL DER LEPRAFÄLLE IN BRASILIEN VON 2010 BIS 2015
}

\section{ORIGINALER ARTIKEL}

MONTEIRO, Lis Mayra Dantas¹, FACCO, Lucas², FECURY, Amanda Alves ${ }^{3}$, ARAÚJO, Maria Helena Mendonça de ${ }^{4}$, OLIVEIRA, Euzébio de ${ }^{5}$, DENDASCK, Carla $V_{\text {Viana }} 6$, SOUZA, Keulle Oliveira da ${ }^{7}$, DIAS, Claudio Alberto Gellis de Mattos ${ }^{8}$

MONTEIRO, Lis Mayra Dantas. Et al. Zahl der Leprafälle in Brasilien von 2010 bis 2015. Revista Científica Multidisciplinar Núcleo do Conhecimento. Jahrgang 05, Ed. 11, Vol. 25, S. 62-70. November 2020. ISSN: 2448-0959, Zugangslink: https://www.nucleodoconhecimento.com.br/gesundheit/leprafaelle-in-brasilien, DOI: 10.32749/nucleodoconhecimento.com.br/gesundheit/leprafaelle-in-brasilien

\section{ZUSAMMENFASSUNG}

Lepra gilt als Infektionskrankheit, die durch das Bakterium Mycobacterium leprae auch bekannt als Hansen es Bacillus verursacht wird. Lepra ist nicht nur eine Krankheit mit allmählicher Evolution, sondern hängt auch von der Beziehung zwischen dem Parasiten und dem Wirt ab. Ziel dieser Studie war es, die Zahl der Leprafälle in Brasilien zwischen 2010 und 2015 in Brasilien aufzuzeigen. Die Forschung wurde bei DATASUS (http://datasus.saude.gov.br/) durchgeführt. Mit dem Voranschreiten der öffentlichen Politik ging die Zahl der Krankheitsfälle in Brasilien

\footnotetext{
${ }^{1}$ Bergbautechnik, als Ergebnis des Bundesinstituts für Amapá (IFAP).

2 Student des Medizinkurses der Föderalen Universität Amapá (UNIFAP).

${ }^{3}$ Biomedizin, PhD in Tropenkrankheiten, Professor und Forscher des Medizinischen Studiengangs der Föderalen Universität Amapá (UNIFAP).

${ }^{4}$ Arzt, Professor und Forscher des Medizinischen Studiengangs der Föderalen Universität Amapá (UNIFAP).

${ }^{5}$ Biologe, PhD in Topische Krankheiten, Professor und Forscher des Leibeserziehungskurses der Föderalen Universität Pará (UFPA).

${ }^{6}$ Theologe, PhD in Psychoanalyse, Forscher am Zentrum für Forschung und Höhere Studien - CEPA.

${ }^{7}$ Soziologe, Master-Student in Anthropischen Studien im Amazonas, Mitglied der Forschungsgruppe "Labor für Bildung, Umwelt und Gesundheit" (LEMAS/UFPA).

${ }^{8}$ Biologe, PhD in Theorie- und Verhaltensforschung, Professor und Forscher des Graduiertenprogramms für berufliche und technologische Bildung (PROFEPT), Bundesinstitut für Amapá (IFAP).
}

RC: 68075

Verfügbar in: https://www.nucleodoconhecimento.com.br/gesundheit/leprafaelle-in- 
zwischen 2010 und 2015 zurück. Es hat eine höhere Prävalenz bei Männern und die höchste Rate von Patienten ist in größeren Altersgruppen, d.h. bei Erwachsenen. Die Inkubationszeit der Krankheit (die zwischen 2 und 7 Jahren liegen kann). Vor diesem Grund können auf individueller Ebene mehrere Probleme ausgelöst werden, wie z. B. schwächende und psychische körperliche Beteiligungen. Um die Zahl der Krankheitsfälle im Land zu reduzieren und die Diagnose so früh wie möglich stellen zu können, sollten die klinischen Befunde und die für diesen Nachweis verfügbaren Tests analysiert werden.

Schlüsselwörter: Epidemiologie, Lepra, Mycobacterium leprae.

\section{EINFÜHRUNG}

Lepra gilt als Infektionskrankheit, weil sie direkten Kontakt mit dem Bacillus benötigt, um zwischen Menschen übertragen zu werden. Es wird durch das Bakterium Mycobacterium leprae auch bekannt als Hansen bacillus verursacht. Diese Krankheit wird nur im Durchschnitt zwischen 2 und 7 Jahren Inkubation erkannt und Symptome treten in der Regel von 11 bis 16 Tagen auf. In dieser symptomatischen Phase ist eine zwischenmenschliche Übertragung möglich, und in der Regel erkrankt nur ein kleiner Teil (BRASIL, 2002; OLIVEIRA et al., 2016).

Lepra kann in zwei Formen klassifiziert werden: Paucibacillary Lepra oder Multibacillary Lepra. In der paucibazillär-Variante hat der Patient eine Resistenz gegen Bazillen und weist bis zu fünf Hautläsionen auf. Bacilli sind im Körper in unterzählige und sind nicht ausreichend für die Übertragung auf andere Menschen. In diesem Fall besteht die Möglichkeit der spontanen Heilung. Multibacillary Lepra ist Teil der sogenannten Sendegruppe, mit sechs oder mehr Hautläsionen. Die Kranken stellen keine Verteidigung gegen das Bakterium dar und beseitigen sie durch die Atemwege (BRASIL, 2002; BRASIL, 2016). Ansteckung erfolgt in der Regel durch direkten Kontakt zwischen einer anfälligen Person und einem unbehandelten Patienten, in der Regel ein Familienmitglied (BRASIL, 2015).

$\mathrm{RC}: 68075$

Verfügbar in: https://www.nucleodoconhecimento.com.br/gesundheit/leprafaelle-in- 
Lepra ist nicht nur eine Krankheit mit allmählicher Evolution, sondern hängt auch von der Beziehung zwischen dem Parasiten und dem Wirt ab. Wenn der Wirt ein effizientes Abwehrsystem hat, neigt es dazu, die Krankheit nicht zu manifestieren. Aus verschiedenen Gründen wie der sozioökonomischen Verwundbarkeit und dem Zusammenleben mit vielen Menschen in der gleichen Umgebung kann es Personen unterschiedlichen Alters und beiderlei Geschlechts erreichen (BRASIL, 2002).

Symptome können auf der Haut oder dem Nervensystem auftreten. Auf der Haut sind sie in der Regel durch Läsionen mit Verlust oder erhöhter Empfindlichkeit gekennzeichnet. Pigmentflecken, Plaques, Infiltrationen, Knollen und/oder Knötchen können auftreten. Im Nervensystem können Symptome als körperliche Behinderungen auftreten und, je nach Grad der Krankheit, Missbildungen in den Extremitäten des Körpers (BRASIL, 2002; FREITAS, 2015).

Die Leprabehandlung erfolgt mit der Kombination mehrerer Medikamente, der sogenannten Polychemotherapie oder PQT. Für Patienten mit der paucibazillär-Form werden zwei Arten von Antibiotika verwendet. Für Personen mit einer multibacillären Form werden drei Arten von Antibiotika empfohlen (BRASIL, 2002; FREITAS, 2015).

Nach Angaben des Gesundheitsministeriums lag die Zahl der neuen Fälle in Brasilien in den untersuchten Jahren bei 193.021 Patienten. (BRASIL, 2017).

\section{ZIEL}

Zeigen Sie die Anzahl der Leprafälle in Brasilien zwischen 2010 und 2015 in Brasilien an.

\section{SYSTEM}

Die Forschung wurde bei DATASUS (http://datasus.saude.gov.br/) durchgeführt, und Lepradaten wurden aus den folgenden Schritten gesammelt: Auf der Registerkarte "Zugriff auf Informationen" wurde "Gesundheitsinformationen (TABNET)" ausgewählt

RC: 68075

Verfügbar in: https://www.nucleodoconhecimento.com.br/gesundheit/leprafaelle-inbrasilien 
und unmittelbar nach einem Klick auf "Epidemiologisch und Morbidität". Auf der nächsten Seite wurde mit dem Klick auf "Leprafälle - Seit 2001 (SINAN)" die Option "Leprosy - seit 2001" gewählt, und im geografischen Bereich haben wir uns für "Brasilien nach Region und Verbandseinheit" entschieden. Die Daten wurden von 2010 bis 2015 gesammelt und wählten: online die Optionen "Diagnosejahr", "Region", "Geschlecht", "Altersgruppe", "Erkennungsmodus", "Diagnosebewertung" und "Hauthaftung". Im Spaltenfeld wurden die Option "Nicht aktiv" und inhaltlich der Ausdruck "Patienten für die Pflege" für die obigen Zeilenoptionen verwendet. Nach der Auswahl wurden diese Daten in das Excel-Tool, die Office-Komponente der Microsoft Corporation, kompiliert. Bibliographische Forschung im Labor des Bundesinstituts für Bildung, Wissenschaft und Technologie von Amapá, Macapá Campus, unter: Rodovia BR 210 KM 3, s/n - Bairro Brasil Novo. CEP: 68.909-398, Macapá, Amapá, Brasil.

\section{ERGEBNISSE}

Abbildung 1 zeigt die Zahl der Leprafälle in Brasilien zwischen 2010 und 2015. Es wird beobachtet, dass in den letzten Jahren die Zahl der Fälle zurückgegangen ist, mit einem leichten Anstieg der Zahl von 2014 im Vergleich zu 2013.

RC: 68075

Verfügbar in: https://www.nucleodoconhecimento.com.br/gesundheit/leprafaelle-in- 
Abbildung 1 Anzahl der Leprafälle in Brasilien zwischen 2010 und 2015.

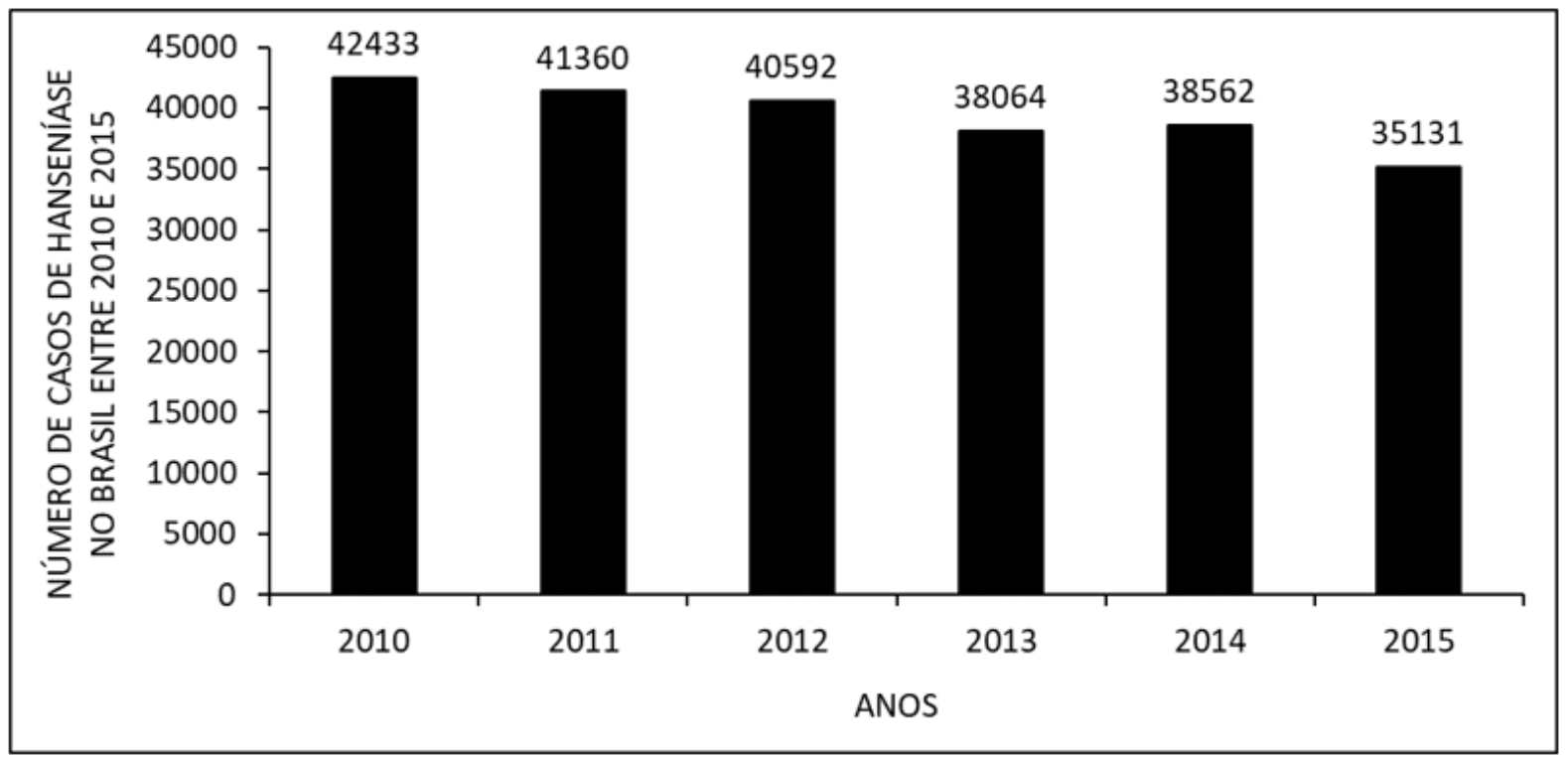

Abbildung 2 zeigt die Zahl der Leprafälle in Brasilien zwischen 2010 und 2015 nach Regionen des Landes. Die Daten zeigen, dass die meisten Besuche im Nordosten und die Minderheit im Süden stattfinden.

RC: 68075

Verfügbar in: https://www.nucleodoconhecimento.com.br/gesundheit/leprafaelle-in- 
Abbildung 2 zeigt die Zahl der Leprafälle in Brasilien zwischen 2010 und 2015 nach Regionen des Landes.

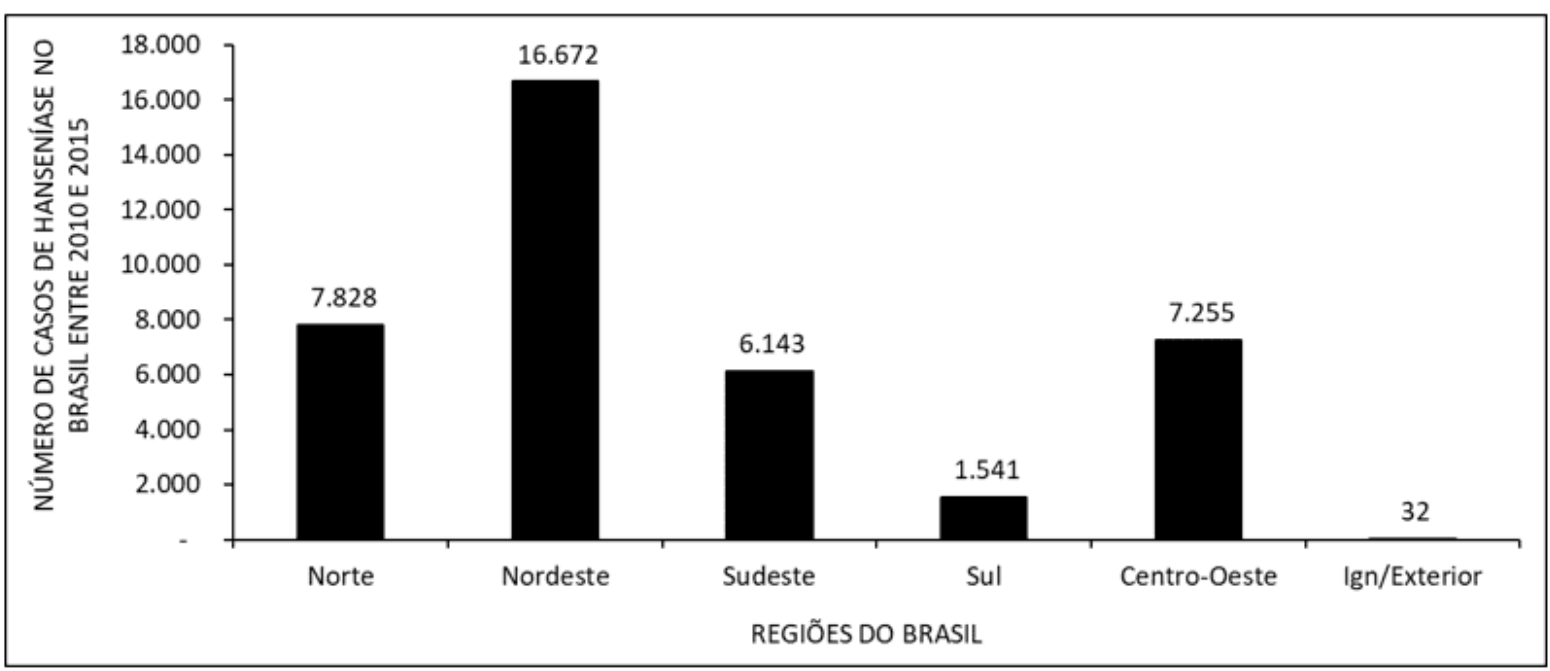

Abbildung 3 zeigt die Zahl der Leprafälle in Brasilien zwischen 2010 und 2015 nach Geschlecht. Wo beobachtet wird, dass es eine höhere Anzahl von Vorkommen bei Männern im Vergleich zu Frauen.

RC: 68075

Verfügbar in: https://www.nucleodoconhecimento.com.br/gesundheit/leprafaelle-in$\underline{\text { brasilien }}$ 
Abbildung 3 zeigt die Zahl der Leprafälle in Brasilien zwischen 2010 und 2015 nach Geschlecht.

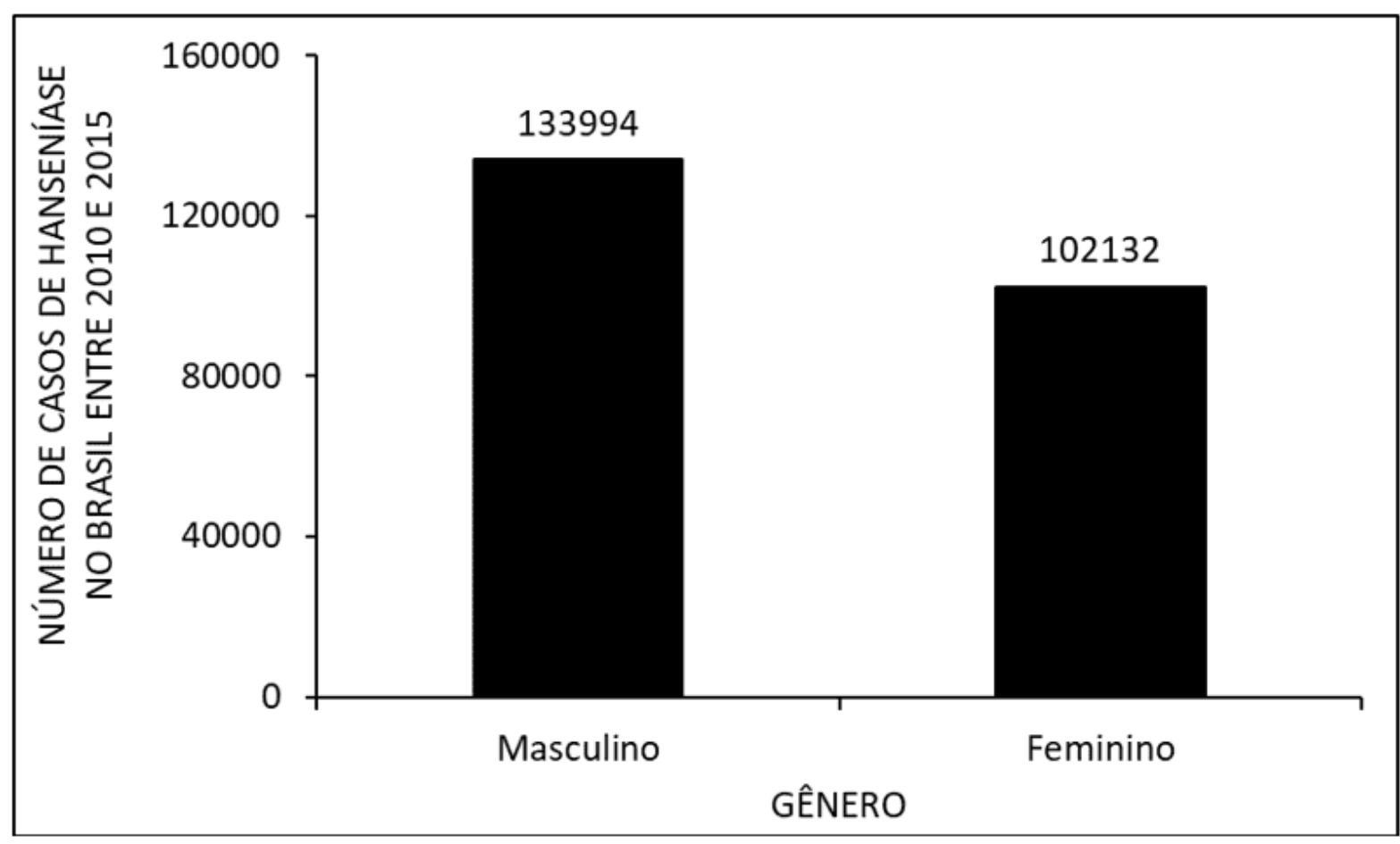

Abbildung 4 zeigt die Zahl der Leprafälle in Brasilien zwischen 2010 und 2015 nach Altersgruppen. Bei Erwachsenen im Alter von 20 bis 64 Jahren und bei Kindern im Alter von 1 bis 9 Jahren und älteren Menschen im Alter von 80 Jahren oder älter ist eine höhere Rate des Auftretens der Krankheit festgestellt.

RC: 68075

Verfügbar in: https://www.nucleodoconhecimento.com.br/gesundheit/leprafaelle-inbrasilien 
Abbildung 4 zeigt die Zahl der Leprafälle in Brasilien zwischen 2010 und 2015 nach Altersgruppen.

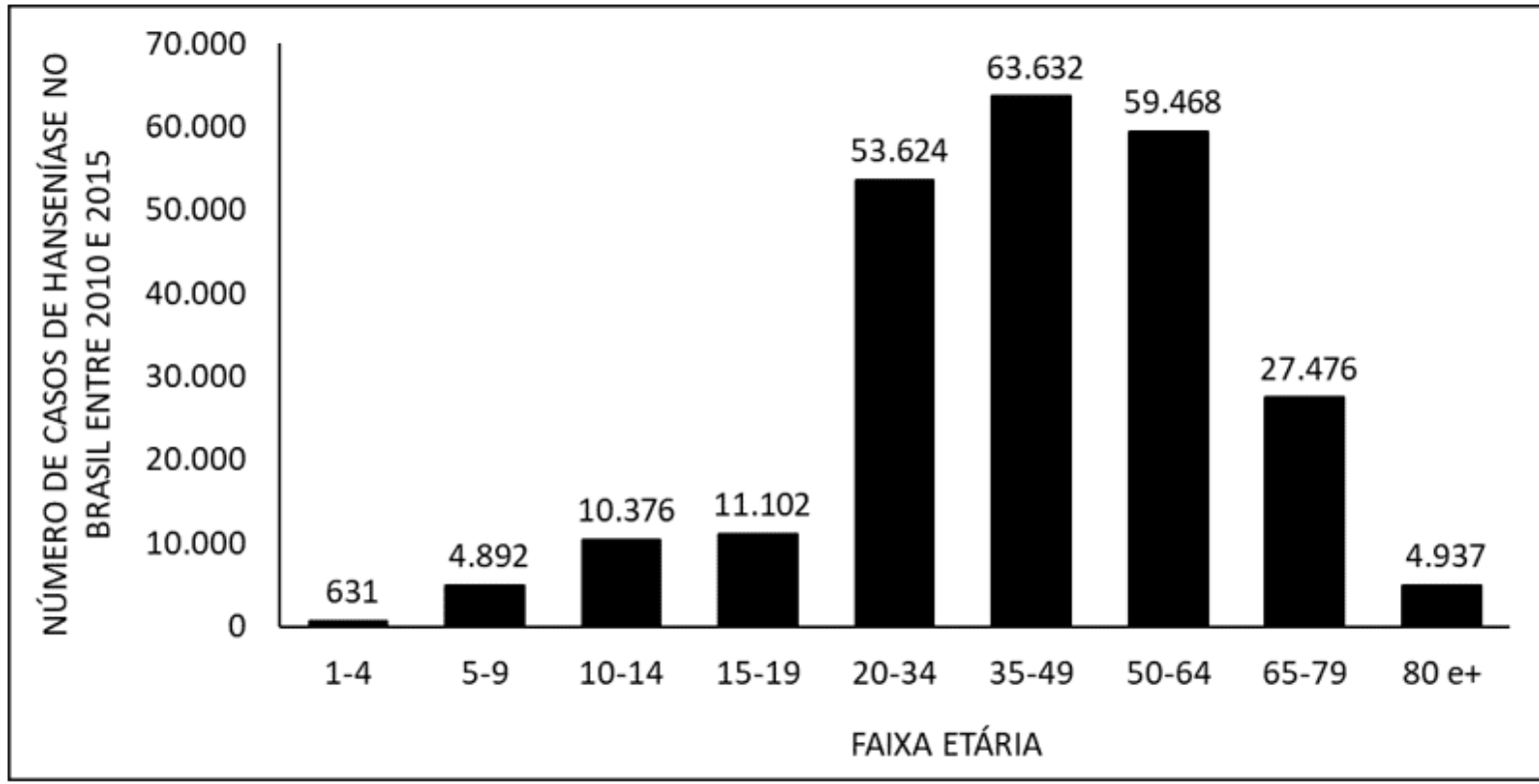

Abbildung 5 zeigt die Anzahl der Leprafälle in Brasilien zwischen 2010 und 2015, je nach Erkennungsmodus. Die Hauptart der Erkennung der Krankheit ist Die Überweisungen, gefolgt von spontaner Nachfrage.

RC: 68075

Verfügbar in: https://www.nucleodoconhecimento.com.br/gesundheit/leprafaelle-inbrasilien 
Abbildung 5 zeigt die Anzahl der Leprafälle in Brasilien zwischen 2010 und 2015, je nach Erkennungsmodus.

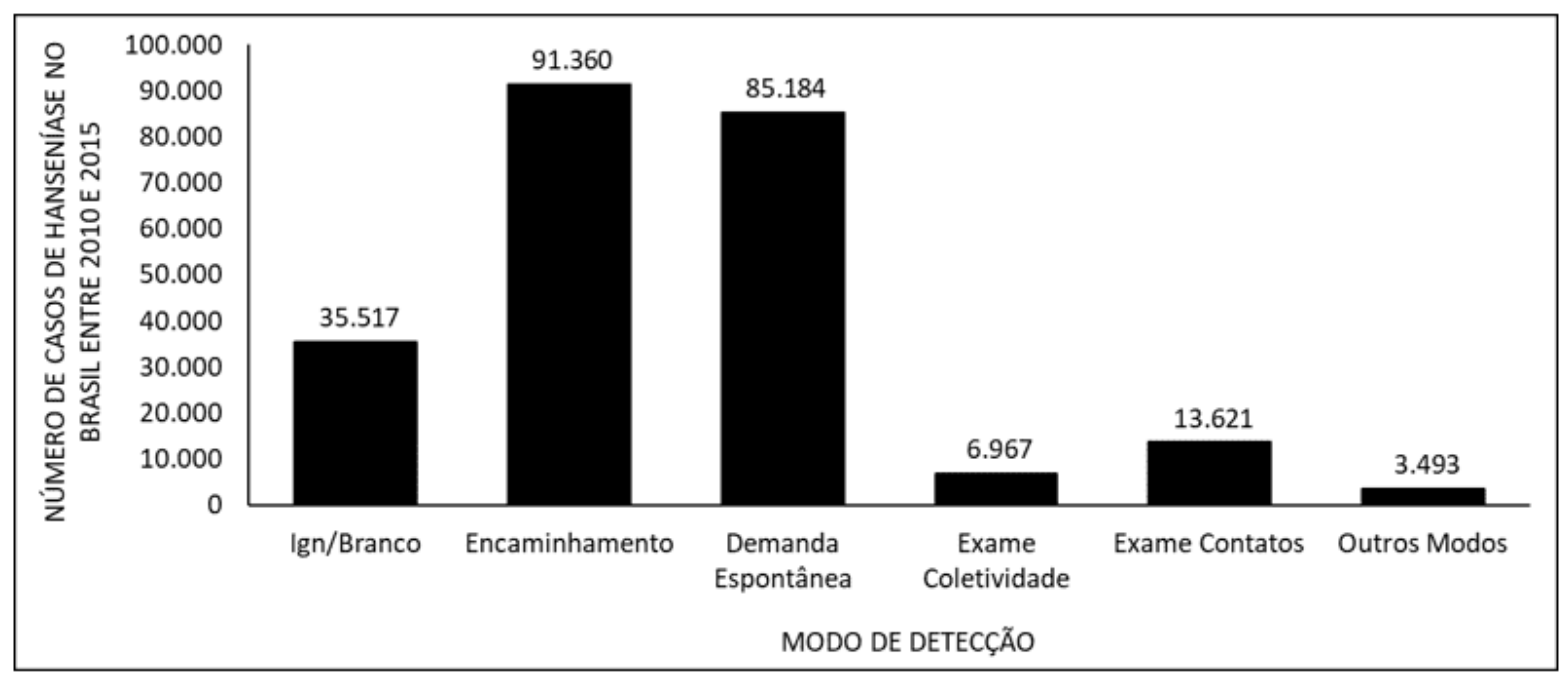

Abbildung 6 zeigt die Zahl der Leprafälle in Brasilien zwischen 2010 und 2015 aufgrund von Hautläsionen. Die Daten zeigen, dass die meisten Patienten 2 bis 5 Hautläsionen haben und Minderheit keine Läsion zeigt.

RC: 68075

Verfügbar in: https://www.nucleodoconhecimento.com.br/gesundheit/leprafaelle-in- 
Abbildung 6 zeigt die Anzahl der Leprafälle in Brasilien zwischen 2010 und 2015 nach Art der Hautläsion.

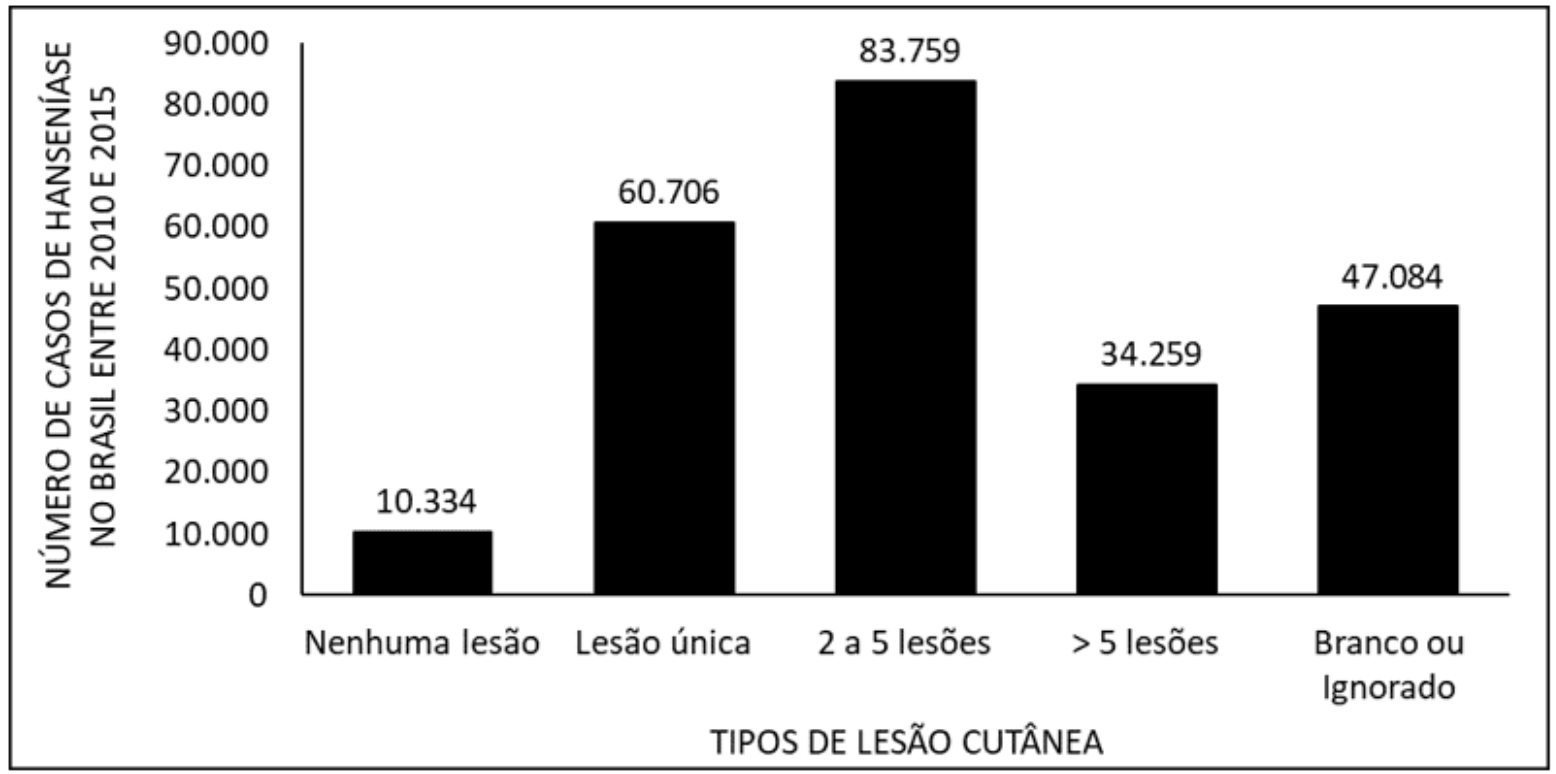

Abbildung 7 zeigt die Zahl der Leprafälle in Brasilien zwischen 2010 und 2015 durch diagnostische Auswertung. Die Daten zeigen, dass die meisten Fälle der Note Null und die Minderheit der Grad II der Krankheit angehören.

RC: 68075

Verfügbar in: https://www.nucleodoconhecimento.com.br/gesundheit/leprafaelle-inbrasilien 
Abbildung 7 zeigt die Zahl der Leprafälle in Brasilien zwischen 2010 und 2015 durch diagnostische Auswertung.

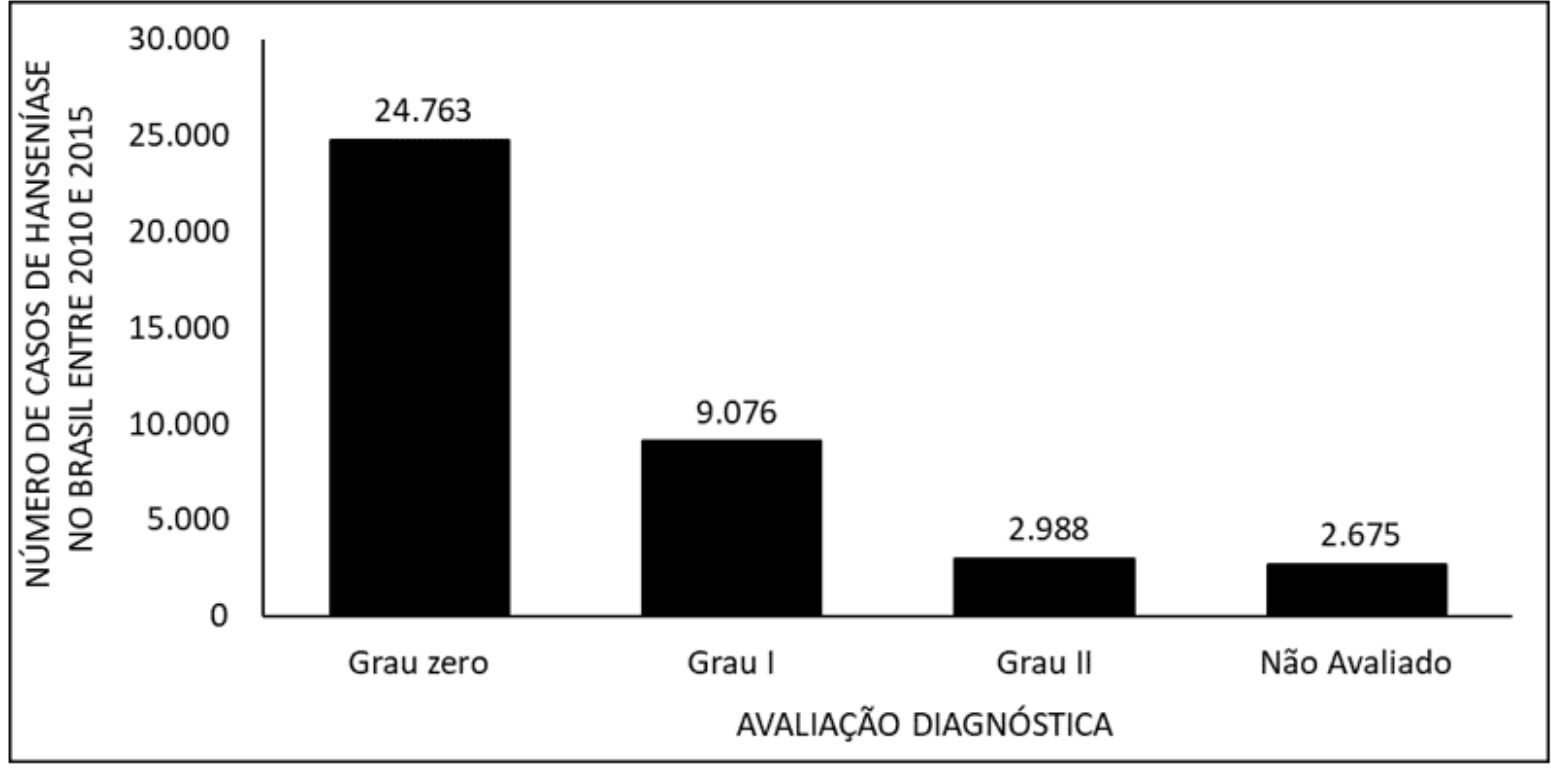

\section{DISKUSSION}

In den Jahren 2010 bis 2015 ging die Zahl der Leprapatienten zurück, 2014 war ein kleiner Anstieg zu verzeichnen (Abbildung 1). Die Daten werden durch die Literatur bestätigt. Der Rückgang ist wahrscheinlich durch die Umsetzung öffentlicher Maßnahmen zur Bewältigung der Krankheit eingetreten, wie eine frühe Diagnose und eine erfolgreiche Behandlung, die zu einer Heilung führt. Der Anstieg der Zahl des Jahres 2014 ist wahrscheinlich auf die Kampagne zur Erkennung neuer Krankheitsfälle zurückzuführen (BRASIL, 2015; 2017; 2017a).

Die Zahl der Leprafälle zeigt mehr Besuche im Nordosten und weniger in der südlichen Region (Abbildung 2). Der Literatur zufolge ist diese hohe Zahl infizierter Menschen im Nordosten wahrscheinlich auf die hohe Übertragungsrate zurückzuführen, die in den Festtagen auftritt. In einer Zeit, in der grundlegende Gesundheitseinheiten nicht handeln. In der südlichen Region bezieht sich die wahrscheinliche Ursache des niedrigen Patientenindex auf die Entfernung zwischen

$\mathrm{RC}: 68075$

Verfügbar in: https://www.nucleodoconhecimento.com.br/gesundheit/leprafaelle-in- 
Gesundheitsdiensten und Dem Patienten, die den Zugang zu einem hohen Grad an körperlicher Behinderung behindert und zur Entwicklung der Krankheit beiträgt (ROCHA, 2015).

Nach Geschlecht hatten Männer in den Jahren 2010 bis 2015 eine höhere Inzidenz der Krankheit als Frauen (Abbildung 3). Beim Vergleich der Daten mit der Literatur wird beobachtet, dass die wahrscheinliche Ursache dieser hohen Rate auf die Tatsache zurückzuführen ist, dass Männer mehr der Krankheit ausgesetzt sind als Frauen, sich Zeit nehmen, um Gesundheitsdienste zu suchen und die Behandlung vor der Heilung aufzugeben. Dies macht ihn anfällig für die Entwicklung der charakteristischen Deformität der Krankheit (ARAGOSO et al., 2014; ARANTES et al., 2010).

Auf der Ebene des Auftretens, Personen der höheren Altersgruppe überwiegen unter den Infizierten. Einer der vorherrschenden Faktoren für diesen Aspekt ist die Inkubationszeit des Bakteriums (Mycobacterium leprae), die sich von zwei bis sieben Jahren erstrecken kann und sich somit im Erwachsenenalter präsentiert und damit in möglichen psychischen Eingriffen gipfelt, da der Patient mit dem Auftreten körperlicher Probleme - verstärkt durch die Verzögerung der Diagnose - nicht in der Lage sein kann, seine berufliche Tätigkeit auszuüben und, daher Schwierigkeiten, einen wirtschaftlichen Beitrag zum familiären Umfeld zu leisten. Darüber hinaus kann bei Familienfällen die Krankheitsgefahr von Kindern erhöht werden (VELÔSO, 2018).

Für die Diagnose von Lepra und deren konsequente Klassifizierung und Inszenierung werden mehrere Aspekte analysiert. Diese Faktoren können je nach verwendeter Klassifikation variieren, von denen zwei die wichtigsten sind, Ridley und Jopling's, basierend auf histopathologischen, klinischen, bakteoskopischen und immunologischen Eigenschaften; Die Klassifikation der Weltgesundheitsorganisation (WHO) verwendet den bakillososkopischen Index in seiner Klassifikation, die festlegt, dass, wenn es maximal fünf Läsionen auf der Kutane-Ebene und nervösen Stamm, paucibazillär Lepra ist. Für den Fall, dass es mehr als fünf Läsionen gibt, auf der

RC: 68075

Verfügbar in: https://www.nucleodoconhecimento.com.br/gesundheit/leprafaelle-in- 
Kutane und nervösen Stamm, gibt es dann multibacillary Lepra. Wenn jedoch sputum abschmiere Mikroskopie beim Patienten verfügbar ist und das positive Ergebnis erreicht wird, basiert die Klassifizierung automatisch auf Multibacillary (LASTÓRIA und ABREU, 2012).

Abhängig von der Manifestation der Lepra können bei der infizierten Person, deren Bazillus aktiv ist, physisch verschiedene Zustände vorhanden sein, einschließlich: granulomatöse Läsionen (die in verschiedenen Formen auftreten können, wie Knötchen oder Papeln), Ödeme, Symptome, die durch Nervenstämme verursacht werden betroffen sind (wie Muskelatrophie, Schwäche und Schmerzen), immunologische Probleme, sensorische und motorische Schwächen, akute und systemische Entzündungsprozesse und andere Auswirkungen auf den Körper (PESSOA, 2019).

Lepra, um keine schwere Beteiligung für den Einzelnen auszulösen, sollte früh diagnostiziert werden, mit der klinischen Logik und den empfohlenen Tests. Wenn die Diagnose zu spät kommt, können die Auswirkungen größer sein. Darüber hinaus sind die Epidemiologie und ihre Kontrolle für eine umfassende Kenntnis der Prävalenzsituation der Krankheit erforderlich, was sich in der Zahl der Fälle widerspiegelt, da, wenn die Kontrolle nicht wirksam ist, eine stärkere Verbreitung der Krankheit und damit eine signifikante Zunahme der Zahl der Fälle auftreten kann. Die Suche nach medizinischer Hilfe im Anfangsstadium der Krankheit gipfelt in der Prävalenz von Fällen der Stufe Null (in denen die Muskelsegmente und ihre jeweiligen Empfindlichkeiten erhalten bleiben und zu einem großen Teil eine gute Prognose des Patienten charakterisieren) (PESSOA, 2019; BRASILIEN, 2018).

\section{FAZIT}

Mit dem Fortschritt der öffentlichen Politik zur Bekämpfung von Lepra, basierend auf der frühdiagnose und Behandlung, ging die Zahl der Krankheitsfälle in Brasilien, in der Mehrheitsstufe, zwischen den Jahren 2010 und 2015 zurück.

$\mathrm{RC}: 68075$

Verfügbar in: https://www.nucleodoconhecimento.com.br/gesundheit/leprafaelle-in- 
Lepra hat eine höhere Prävalenz bei Männern, und die wahrscheinliche Ursache dieser Realität ist die größere Exposition von Männern gegenüber dieser Pathologie, da die Suche nach Gesundheitsdienstleistungen weniger umfassend ist und darüber hinaus die größere Aufgabe der Behandlung, was zum Vordringen der Krankheit und der daraus resultierenden Entstehung organischer Schwächen des Individuums führt.

Darüber hinaus ist die höchste Rate der Patienten in größeren Altersgruppen, d. h. bei Erwachsenen, und der Hauptfaktor ist dafür die Inkubationszeit der Krankheit verantwortlich (die zwischen 2 und 7 Jahren liegen kann). Vor diesem Grund können auf individueller Ebene mehrere Probleme ausgelöst werden, wie z. B. schwächende und psychische körperliche Eingriffe und familiäre, da mit dem Auftreten von Schwäche wirtschaftliche Schwierigkeiten aufgrund des Verlusts bezahlter beruflicher Tätigkeit entstehen können.

Um die Zahl der Krankheitsfälle im Land zu reduzieren und die Diagnose so früh wie möglich stellen zu können, sollten die klinischen Befunde und die für diesen Nachweis verfügbaren Tests analysiert werden. Wenn der Patient seine Diagnose im frühen Stadium der Krankheit stellen kann, gibt es in der Regel eine gute Prognose, und seine organische Gesundheit kann stark erhalten werden, die Empfindlichkeit und Integrität seiner Muskelsegmente.

\section{VERWEISE}

ARAGOSO, I.; CARVALHO, R.M.B.; SOUSA, C.M.; VIEIRA, G.D. Hanseníase em Rondônia: incidência e características dos casos notificados, 2001 a 2012. Epidemiologia e Serviços de Saúde, v.23, n.2, Brasília, jun. 2014.

ARANTES, C.K.; FILIPE, M.S.; GARCIA, M.L.R.; NARDI, M.T.; PASCHOAL, V.D.A. Avaliação dos serviços de saúde em relação ao diagnóstico precoce da hanseníase. Epidemiologia e Serviços de Saúde, v.19, n.2, Brasília, jun. 2010.

$\mathrm{RC}: 68075$

Verfügbar in: https://www.nucleodoconhecimento.com.br/gesundheit/leprafaelle-inbrasilien 
BRASIL. Ministério da Saúde. Secretaria de Políticas de Saúde. Departamento de Atenção Básica. Guia para o controle da hanseníase. $1^{\underline{a}}$ ed., Brasília (DF): Ministério da Saúde, 2002. Disponível em: <http://bvsms.saude.gov.br/bvs/publicacoes/guia_de_hanseniase.pdf> Acesso em: 24 ago 2017.

BRASIL. Ministério da Saúde. Secretaria de Vigilância em Saúde. Departamento de Vigilância das Doenças Transmissíveis. Diretrizes para vigilância, atenção e eliminação da Hanseníase como problema de saúde pública: manual técnicooperacional. 1aㅗ ed., Brasília (DF): Ministério da Saúde, 2016. Disponível em: $<$ http://www.saude.pr.gov.br/arquivos/File/DiretrizesdoManuaTcnicoOperacionaldeH ansenase.pdf> Acesso em: 05 set 2017.

BRASIL. Ministério da Saúde. Secretaria de Vigilância em Saúde. Departamento de Vigilância das Doenças Transmissíveis. Eliminar a hanseníase é possível: um guia para os municípios. Brasília (DF): Ministério da Saúde, 2015. Disponível em: $<$ http://bvsms.saude.gov.br/bvs/publicacoes/eliminar_hanseniase_possivel_versao_p reliminar.pdf> Acesso em: 05 set 2017.

BRASIL. Boletim Epidemiológico. Brasilía DF. Ministério da Saúde. 49, n. 4, 12p. 2018.

FREITAS, A.A. Resposta imune celular e humoral a proteínas recombinantes do Mycobacterium leprae em pacientes com outras dermatoses. Tese (Doutorado) Universidade Federal de Goiás, Instituto de Patologia Tropical e Saúde Pública, Program de Pós Graduação em Medicina Tropical e Saúde Pública, Goiânia, 2015. Disponível em: $<\mathrm{https}: / /$ posstrictosensu.iptsp.ufg.br/up/59/o/AlinedeAraujoFreitas_2015_vers\%C3\%A3ofinal. pdf> Acesso em: 12 set 2017.

RC: 68075

Verfügbar in: https://www.nucleodoconhecimento.com.br/gesundheit/leprafaelle-inbrasilien 
LASTÓRIA, J. C; ABREU, M. A. M. M. Hanseníase: diagnóstico e tratamento. Diagn Tratamento, v. 17, n. 4, p. 173-179, 2012.

OLIVEIRA, Ciane Martins de; et. al. A evolução da assistência ao paciente com Hanseníase: dos leprosários à poliquimioterapia. Revista Científica Multidisciplinar Núcleo do Conhecimento. Ano 01, Vol. 06, Ed. 08, pp. 68-80. Agosto de 2016. ISSN: 2448-0959

PESSOA, M. M. S. F. S. Hanseníase no Brasil: Uma revisão literária, nos anos de 2014 a 2019. 45p. Trabalho de Conclusão de Curso (Graduação de Farmácia) Universidade Federal do Rio Grande do Norte, Rio Grande do Norte, Natal, 2019.

ROCHA, A.C.A.A. Análise da sazonalidade da incidência de hanseníase segundo regiões geográficas, climas e biomas do Brasil. 2015. 82 f. Dissertação (Mestrado em Ciências Ambientais) - Universidade do Estado de Mato Grosso, Programa de Pós-Graduação em Ciências Ambientais, Cáceres/MT.

VELÔSO, D. S. Perfil clínico-epidemiológico da hanseníase no estado do Piauí, no período de 2009 a 2016. 127p. Dissertação (Mestrado em Medicina Tropical) Instituto Oswaldo Cruz, Piauí, Teresina, 2018.

Eingereicht: November 2020.

Genehmigt: November 2020.

RC: 68075

Verfügbar in: https://www.nucleodoconhecimento.com.br/gesundheit/leprafaelle-in- 